\title{
COMMON INFECTING BACTERIA AND ANTIBIOTIC SENSITIVITY PATTERN IN PATIENTS WITH SEVERE LOWER RESPIRATORY TRACT INFECTIONS AT A TERTIARY CARE HOSPITAL
}

\author{
Ramya Sudha Chittoor ${ }^{1}$, Lokeswara Reddy Avula2 ${ }^{2}$ Aruna Gorthi ${ }^{3}$ \\ 1 Junior Resident, Department of Pulmonary Medicine, SVRR Government General Hospital, Sri Venkateshwaraa Medical College, \\ Tirupati, Andhra Pradesh. \\ ${ }^{2}$ Senior Resident, Department of Pulmonary Medicine, SVRR Government General Hospital, Sri Venkateshwaraa Medical College, \\ Tirupati, Andhra Pradesh. \\ ${ }^{3}$ Professor, Department of Pulmonary Medicine, SVRR Government General Hospital, Sri Venkateshwaraa Medical College, Tirupati, \\ Andhra Pradesh.
}

ABSTRACT

\section{BACKGROUND}

Lower respiratory tract infections are the commonest infectious diseases affecting humans worldwide. They are considered a major public health problem and cause morbidity and mortality. Acute and chronic bronchitis, influenza, pneumonia, lung abscess and infective exacerbations of bronchiectasis are the common lower respiratory infections that present at tertiary care hospitals. In this study, we are considering pneumonia, lung abscess and infective exacerbations of bronchiectasis as they are of bacterial origin and culture and sensitivity and isolation is easier at our institute, viral assays are not available. This study was started to understand the common bacteria isolated in patients with severe lower respiratory tract infections and the antibiotic sensitivity pattern in these patients.

\section{MATERIALS AND METHODS}

All patients with severe lower respiratory tract infections - Pneumonia, Lung abscess and Bronchiectasis who presented to Chest OP and Emergency Department of SVRR Government General Hospital, Tirupati, Andhra Pradesh were recruited to the study. Diagnosis was made by detailed history taking, Chest x-ray, CT scan of chest where necessary, Sputum Gram Staining and Pyogenic culture. Empirical antibiotics were started and later antibiotics were changed based on susceptibility pattern.

\section{RESULTS}

Out of 100 patients recruited to the study, 64 were male and 36 were female. Pneumonia was seen in 58 patients, Bronchiectasis in 32 patients, Lung abscess in 10 patients. Most common organisms isolated were Klebsiella, Pneumococci, Staphylococcus aureus, H. Influenza, E. coli, Pseudomonas, M. catarrhalis in that order. Commonest comorbidity was Diabetes (38 cases). Pneumonia patients with 9.8 days admission had the shortest hospital stay. All patients recovered and there were no deaths.

\section{CONCLUSION}

Male patients (64\%) are more prone for Serious LRTI due to Alcoholism and smoking and are commonly infected with Klebsiella and Pneumococci. Female patients (36\%) are infected more commonly with H. Influenza, Klebsiella and E. coli and showed higher incidence of anaemia and Diabetes. Pneumonia when managed meticulously has a very good prognosis.

\section{KEYWORDS}

Severe Lower Respiratory Infections, Infecting Bacteria, Antibiotic Sensitivity Pattern, Prognosis.

HOW TO CITE THIS ARTICLE: Chittoor RS, Avula LR, Gorthi A. Common infecting bacteria and antibiotic sensitivity pattern in patients with severe lower respiratory tract infections at a tertiary care hospital. J. Evolution Med. Dent. Sci. 2017;6(73):51905194, DOI: $10.14260 /$ Jemds/2017/1128

\section{BACKGROUND}

Lower respiratory tract infections are a persistent and pervasive public health problem. They cause a greater burden of disease world-wide than Human Immunodeficiency Virus, malaria, cancer or heart attack. ${ }^{1}$ The lower respiratory tract starts at the level of larynx, includes the tracheobronchial tree and ends at the level of the air exchanging alveoli. ${ }^{2}$ Lower Respiratory Tract Infections (LRTIs) are the most common infectious diseases affecting humans world-wide.

Financial or Other, Competing Interest: None.

Submission 03-05-2017, Peer Review 08-06-2017,

Acceptance 14-06-2017, Published 11-09-2017.

Corresponding Author:

Dr. Lokeswara Reddy Avula,

Kummarapalli (Vill) \& (Post),

Renigunta (Mandal),

Chittoor-517520, Andhra Pradesh.

E-mail: loka.mbbs@gmail.com

DOI: $10.14260 /$ jemds $/ 2017 / 1128$

(c) (i) () $९$
Despite being the cause of significant morbidity and mortality, LRTIs are often mistreated and underestimated. ${ }^{3}$ The commonly encountered severe LRTIs are Influenza (A and H1NI), Pneumonia, Bronchiectasis, Lung abscess and Bronchitis. Children tend to get more respiratory infections than adults because of less immunity built up to many viruses that can cause these infections. Acute lower respiratory infections are a leading cause of sickness and mortality both in children and adults.

Pneumonia is the commonest cause of death in children below 5 years and often the final disease to occur before death in geriatric patients. ${ }^{4}$ Pneumonia is defined as an acute respiratory illness associated with recently developed radiological pulmonary shadowing which may be segmental, lobar or multilobar. It occurs about five times more frequently in the developing world than the developed world. The incidence of community-acquired pneumonia (CAP) ranges from 4 million to 5 million cases per year, with $25 \%$ 
requiring hospitalisation. The problem is much greater in the developing countries where pneumonia is the most common cause of hospital attendance in adults. Pneumonias are usually classified as community-acquired pneumonia, hospital-acquired pneumonia and those occurring in immunocompromised host or patient with underlying damaged lung including suppurative and aspiration pneumonia. It is classified as either community acquired or hospital acquired depending on where the patient contracted the infection. It is life threatening in the elderly or those who are immunocompromised. In 2015, there were about 291 million cases of pneumonia worldwide. These resulted in 2.74 million deaths down from 3.4 million deaths in $1990.4 .8 \%$ of all deaths in 2013 were pneumonia deaths. It is the leading cause of death in children less than 5 years. The most common cause of pneumonia is Pneumococcus which accounts for two-thirds of pneumonia. This is a dangerous type of lung infection with a mortality rate of $25 \%$. Other infections like parasitic infections, Cryptosporidiosis, adenovirus, influenza and parainfluenza can also cause pneumonia. In cases of pneumonia, high grade fever, productive cough with blood stained sputum, signs of lobar consolidation and neutrophilia are highly suggestive of Streptococcus pneumonia infection. A dry cough fever with diffuse infiltrates on chest $\mathrm{x}$-ray and extrapulmonary symptoms are indicative of Mycoplasma pneumonia, Chlamydia pneumonia or Legionella Pneumonia. The best approach to select a good initial empirical antibiotic is to identify the risk factors contributing to morbidity and mortality and then select empirical therapy accordingly. The most important predictors of patient morbidity and mortality are age, underlying disease and severity of illness. Patients with Pneumonia who survive in hospital may still experience adverse outcomes after discharge including readmission and death due to relapse of Pneumonia. Early readmission rates range from $8-46 \%$ and the mortality rate within 90 days after discharge can be as high as $14 \%$. Even at 1 year, mortality is considerably higher after hospitalisation, with a significant increase in risk of cardiovascular events.

Lung abscess is a type of liquefactive necrosis of the lung tissue and formation of cavities (more than $2 \mathrm{~cm}$ ) containing necrotic debris or fluid caused by microbial infection. It can be caused by aspiration, which may occur during altered consciousness and it usually causes a pus-filled cavity. Lung abscess is considered primary (60\%) when it results from existing lung parenchymal process and is termed secondary when it complicates another process, e.g., vascular emboli or follows rupture of extrapulmonary abscess into lung. Lung abscess is now rarely seen in the developed world but in a country like ours, it is commonly diagnosed. It has a high mortality of $20-30 \%$ and is most commonly seen in alcoholic men more than 50 years. ${ }^{5}$

Bronchiectasis is today considered an "Orphan Disease" seen only in the developing world, and evokes little commercial interest. Bronchiectasis is an abnormal, chronic enlargement of the bronchi, the passageways from the trachea to the alveoli that are the air-exchanging parts of the lungs. Bronchiectasis generally occurs as a result of infection, although non-infectious factors may contribute to the development of this condition. Accompanying the enlargement of the bronchi is their decreased ability to clear secretions. Failure to clear secretions allows microbes and particles to collect in them, which leads to more secretions and inflammation that further damage the airways, causing more dilation in a vicious cycle. Bronchiectasis may occur in a single portion of the lung (Localised) or throughout the lungs (Diffuse) and is the major lung abnormality of cystic fibrosis. It may have several different contributing factors, such as abnormal cilia, and its course may vary greatly from causing no symptoms to causing death. It presents itself as repeated remissions and exacerbations of severe cough, copious amounts of mostly foul-smelling sputum, haemoptysis on and off, (Sometimes life threatening) along with breathlessness. In most patients, symptoms are present since childhood, though some patients present in middle age. When the disease is extensive, cor pulmonale and respiratory failure occur early in life. It is not curable. The goal of treatment is to control secretions and minimise the risk of infections. Chest physiotherapy and postural drainage play a major role in the management. Surgery gives good results when the disease is localised. Lobectomy and Pneumonectomy are considered in unilateral disease.

Acute bronchitis is defined as an acute illness that occurs in previously asymptomatic younger individuals with severe cold which seems to have "descended" into the chest presenting as severe cough and sometimes breathlessness and wheeze with no history of asthma or sinusitis. These episodes are mostly of viral origin but sometimes the aetiological agents are Gram-positive organisms like Streptococcus pneumonia and Staphylococcus aureus and Gram-negative organisms like Haemophilus influenza, Klebsiella pneumonia, Pseudomonas and Acinetobacter.

Influenza, both of types A and H1N1 (Swine flu) usually occur as annual epidemics and occasionally pandemics. The most recent pandemic was in 2009 and there were about 6000 deaths world over. Even in India, about 1100 patients succumbed to the H1N1 virus. Rates of infection are highest among children, with severe illness and deaths occurring in patients $<2$ years and $>65$ years. Underlying medical conditions that increase the risk of hospitalisation include Diabetes, Cardiovascular disease, chronic respiratory disease like Bronchial asthma and Pregnancy. Symptoms include severe cold and sore throat which in immunocompetent individuals, soon resolve. However, in patients with the abovementioned medical conditions, it rapidly progresses to Pneumonia, ARDS and Respiratory failure. Extremely good supportive care is necessary besides antivirals like Oseltamivir. The patient has to be isolated to prevent spread through droplets and fomites.

The common aetiological agents in patients with Pneumonia, Lung abscess and Bronchiectasis are Grampositive organisms like Streptococcus pneumonia and Staphylococcus aureus and Gram-negative organisms like Haemophilus influenza, Klebsiella pneumonia, Pseudomonas and Acinetobacter. ${ }^{6}$

In this study, an attempt is being made to know about the common bacterial organisms causing severe LRTIs and to learn about their susceptibility patterns in patients admitted to the Chest Wards of a tertiary care hospital and to learn about their prognosis. 


\section{MATERIALS AND METHODS}

Study Design

Hospital-based cross-sectional study.

\section{Study Setting}

Chest wards and RICU of Department of Pulmonary Medicine, SVRR Govt. General Hospital, Tirupati.

\section{Period of Study}

May $1^{\text {st }} 2016$ to Sep $1^{\text {st }} 2016$ (4 months).

\section{Sample Size}

Total number of patients admitted with severe LRTIs (Pneumonia, Bronchiectasis or Lung abscess) in the Chest wards and RICU of Department of Pulmonary Medicine during the study period i.e. 100 patients.

\section{Inclusion Criteria}

1. Patients with severe LRTI and more than 18 years of age.

2. Patients willing to participate in the study.

\section{Exclusion Criteria}

1. Severe LRTI patients with age less than 18 years.

2. Patients whose sputum or chest x-ray were suggestive of tuberculosis.

3. Patients not willing to participate in the study.

\section{Methodology}

100 patients whose history, clinical and radiological findings were consistent with Pneumonia, Bronchiectasis or Lung abscess were recruited to the study and admitted in the Chest or RICU wards of Department of Pulmonary Medicine, SVRR Govt. General Hospital, Tirupati. Antibiotics were started empirically after the sputum was sent for Gram staining, AFB staining and pyogenic culture. AFB-positive patients were excluded from the study. The initial route of therapy depended on the severity of illness, the ability of the patient to tolerate oral medication and the likeliness of patient compliance. If the initial treatment was parenteral, shift to oral therapy was considered, once the clinical condition improved and the patient could tolerate oral fluids and had a temperature of less than $100^{\circ} \mathrm{F}$ for at least 48 hours. The choice of drug was made on known susceptibility pattern if the causative organism had been identified or from the list of empirical drugs. The routinely used empirical antibiotics at our centre are Inj. Amoxiclav, Inj. Amikacin and Inj. Metronidazole. Later antibiotics were changed based on the obtained culture results. After 5-7 days of injectables and clinical and radiological improvement, the patients were discharged with step down to oral antibiotics and followed up after 7-10 days.

\section{RESULTS}

Out of 100 patients admitted with severe LRTI, 64 were male and 36 were female.

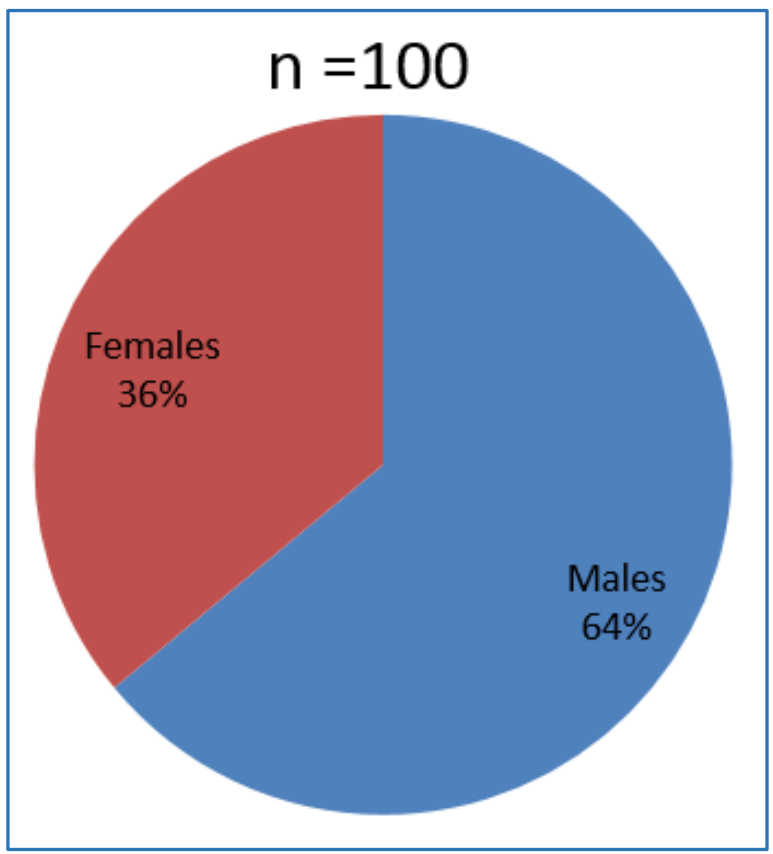

\section{Sex Distribution}

Most of the patients were of the age group 51-70 years (42\%).

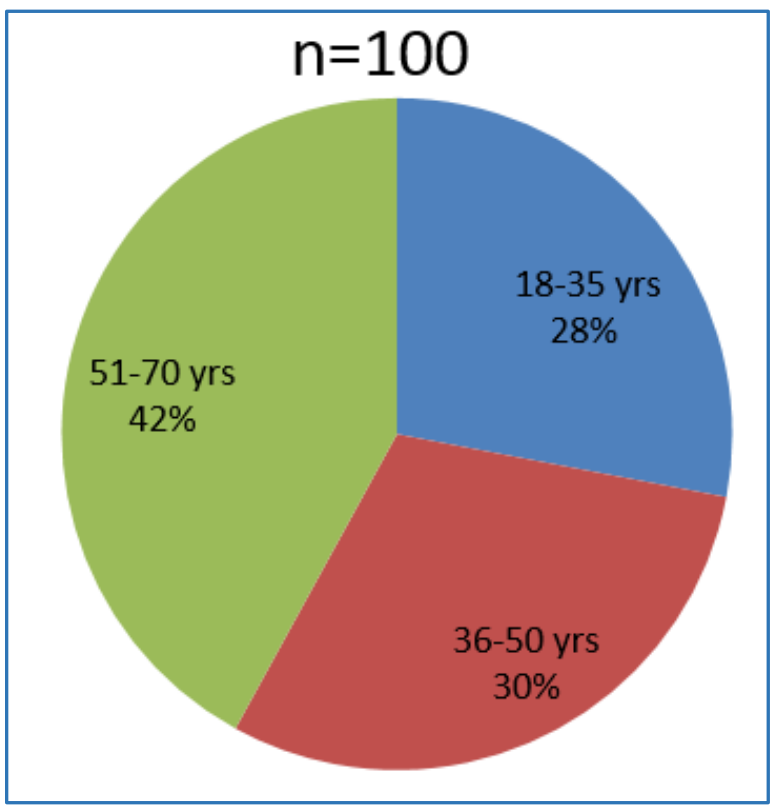

\section{Age Distribution}

Out of the 100 patients, 58 were Pneumonia, 32 were Bronchiectasis and 10 were Lung abscess cases.

\begin{tabular}{|c|c|}
\hline Type of LRTI & No. of Patients \\
\hline Pneumonia & 58 \\
\hline Bronchiectasis & 32 \\
\hline Lung abscess & 10 \\
\hline \multicolumn{2}{|c|}{ Table 1. Types of LRTI } \\
\hline
\end{tabular}


Most of the males were smokers (54\%) and Diabetes was the commonest type comorbidity (38\%). Some patients of LRTI had overlapping comorbidities.

\begin{tabular}{|c|c|}
\hline Comorbidity & \% \\
\hline Smokers & 54 \\
\hline Alcoholism & 22 \\
\hline Diabetes & 38 \\
\hline CKD & 12 \\
\hline Table 2. Comorbidities \\
\hline
\end{tabular}

The most common organisms isolated in order of frequency were Klebsiella pneumonia (36\%) and least common were Moraxella catarrhalis (2\%).

\begin{tabular}{|c|c|}
\hline Organism & \% \\
\hline Klebsiella & 36 \\
\hline Pneumococci & 24 \\
\hline H. influenza & 14 \\
\hline E. coli & 10 \\
\hline Staph. aureus & 8 \\
\hline Pseudomonas & 6 \\
\hline M. catarrhalis & 2 \\
\hline \multicolumn{2}{|c|}{ Table 3. Organisms Isolated } \\
\hline
\end{tabular}

The commonest organisms isolated in the 58 Pneumonia patients were Pneumococci (52\%) followed by Klebsiella (26\%) and Haemophilus (22\%). In the 32 cases of Bronchiectasis, the infecting organisms were Klebsiella $(60 \%)$ followed by Pseudomonas (32\%) and Staphylococcus aureus (6\%) and Moraxella (2\%). The 10 patients with Lung abscess showed more than 2 organisms including Klebsiella, E. coli, Pseudomonas and Staphylococcus.

\begin{tabular}{|c|c|}
\hline Type of LRTI & Organism Isolated \\
\hline \multirow{3}{*}{ Pneumonia } & Pneumococci $-52 \%$ \\
\hline & Klebsiella (26\%) \\
\hline & Haemophilus (22\%) \\
\hline \multirow{3}{*}{ Bronchiectasis } & Klebsiella (60\%) \\
\hline & Pseudomonas (32\%) \\
\hline & Staphylococcus aureus (6\%) Moraxella (2\%) \\
\hline Lung abscess & $\begin{array}{l}\text { Klebsiella, E. coli, Pseudomonas and } \\
\text { Staphylococcus }\end{array}$ \\
\hline Table 4. T & of LRTI vs. Type of Organism Isolated \\
\hline
\end{tabular}

Almost all the isolated organisms were sensitive to Levofloxacin and Piperacillin-Tazobactam.

\begin{tabular}{|c|c|c|}
\hline Organism & Commonly Sensitive to & \% Sensitivity \\
\hline \multirow{2}{*}{ Klebsiella } & Levofloxacin & 68 \\
& (Piperacillin + Tazobactam) & 32 \\
\hline \multirow{2}{*}{ Pneumococci } & Amoxi-clav & 72 \\
& Azithromycin. & 28 \\
\hline \multirow{2}{*}{ Staph. aureus } & Amoxi-clav & 50 \\
& Ceftriaxone & 50 \\
\hline \multirow{2}{*}{ H. influenza } & Ciprofloxacin & 61 \\
& Gentamycin & 39 \\
\hline \multirow{2}{*}{ E. coli } & Gentamycin & 75 \\
& Azithromycin & 25 \\
\hline \multirow{2}{*}{ Pseudomonas } & Levofloxacin & 81 \\
& Ceftazidime & 19 \\
\hline \multirow{2}{*}{ M. catarrhalis } & Amoxi-clav & 55 \\
& \multicolumn{2}{|c|}{ Levofloxacin } \\
\hline \multicolumn{2}{|c|}{ Table 5. Organism Sensitivity Pattern } \\
\hline
\end{tabular}

Average duration of admission for Pneumonia was 9.8 days, Bronchiectasis was 13.4 days and for Lung abscess was 12.4 days.

\begin{tabular}{|c|c|}
\hline Type of LRTI & Avg. Days of Admission \\
\hline Pneumonia & 9.8 \\
\hline Bronchiectasis & 13.4 \\
\hline Lung abscess & 12.4 \\
\hline \multicolumn{2}{|c|}{ Table 6. Clinical Course of the Disease } \\
\hline
\end{tabular}

Smokers and alcoholics had a longer duration of stay in hospital. Even patients with diabetes and CKD had a longer duration of stay.

\begin{tabular}{|c|c|c|c|}
\hline Comorbidity & $\begin{array}{c}\text { Days in } \\
\text { Hospital }\end{array}$ & $\begin{array}{c}\text { Days in } \\
\text { Hospital }\end{array}$ \\
\hline Smokers & 13.6 & Non-Smokers & 9.6 \\
\hline Alcoholics & 12.9 & Non-alcoholics & 11.4 \\
\hline DM & 14.1 & Non-DM & 8.4 \\
\hline CKD & 13.1 & Non-CKD & 11.4 \\
\hline Table 7. Comorbidities and Duration of Hospital Stay \\
\hline
\end{tabular}

\section{DISCUSSION}

In a developing country like India, severe LRTIs follow a complicated course and management is often difficult due to the problems associated with the identification of aetiological agents and the administration of appropriate treatment in cases requiring antibiotic therapy.7.Hence, this study is justified.

Our study showed more males than females (64:36). This could be due to high incidence of smoking and alcoholism in men. Most of the patients were in 51-71 years of age group which may be due to comorbidities being more seen in this age group.

Diabetes was the commonest comorbid disease (38\%) in our study.

Aetiological agents of LRTIs cannot be determined clinically and differ from area to area. ${ }^{8}$ In our study, Klebsiella was the commonest isolated organism (36\%), followed by Streptococcus pneumonia (24\%) and Haemophilus influenza (14\%).

Antibiotics are the cornerstone of treatment in severe LRTIs. When an antibiotic is required, the choice of drug, as earlier stated will depend on the severity of illness, age of the patient, the presence of any other underlying disease, history of drug reactions and the likely compliance of the patients. Amoxicillin and Doxycycline are routinely the best drugs recommended as initial therapy and are effective against most causative bacteria, though Amoxicillin is not effective against beta-lactamase producing organisms. Moraxella catarrhalis produces a beta-lactamase. About 20\% of Haemophilus influenza also produce beta-lactamase. If the clinical response is slow or the patient has severe exacerbation, cefaclor or Amoxicillin-Potassium Clavulanate are the best choice.

In or study, all the isolated organisms were sensitive to Levofloxacin and Piperacillin-Tazobactam. Pneumococci and Staphylococci aureus showed sensitivity in addition to Amoxi-clav, Azithromycin and Ceftriaxone. Haemophilus influenza and Escherichia coli showed sensitivity to Gentamycin.

Longest duration of hospital stay was for Bronchiectasis (13.4\%) and least was for Pneumonia (9.8\%). 
When compared to non-smokers and non-alcoholics, smokers and alcoholism had to stay longer in the hospital. Diabetes and renal failure patients also had to stay longer in the hospital.

The risk of death from LRTIs increases with age. In 2015, in Europe, case fatality rate of $5.6 \%$ in those less than 65 years and $47.2 \%$ in those more than 85 years has been stated. In another study, 12-fold greater likelihood of death within 30 days of hospital admission was seen in patients more than 85 years as compared to those less than 65 years. In cases of Pneumonia, risk factors for death include age $>65$ years, ICU admission, female sex, multiple organisms, pleural effusion, atypical pneumonia, hospital-acquired pneumonia, acute renal failure, multilobar involvement and shock. The risk of pneumonia related mortality increases three fold if pneumonia is due to Pneumococcus (6-40\%). There were no deaths in our study and all the patients were followed up at the Chest OPD after 10 days.

The increasing number of antibiotic-resistant bacteria is a worrying threat to the global population and a critical challenge for the health care sector. Data show that worldwide almost $10 \%$ of Streptococcus pneumonia strains are resistant to penicillin and macrolides. Nowadays, difficult to treat bacteria Methicillin-resistant Staphylococcus aureus and multidrug resistant Klebsiella and Pseudomonas are emerging.

\section{CONCLUSION}

1. Greater number of males in our study shows that men are more prone to severe LRTIs probably because of alcoholism and smoking.

2. Males appeared to be more commonly infected with Klebsiella and Pneumococci.

3. Females showed a higher incidence of anaemia and were more commonly infected with H. Influenza, Klebsiella and E. coli.

4. Patients with Diabetes, CKD, alcoholics and smokers had to stay longer in the hospital.

\section{Prevention Strategies and Future Development}

Protection people by immunisation against infections is a great step. Though there are vaccines against Pneumococci and Influenza viruses, currently there is no immunisation against Respiratory Syncytial Virus (RSV).
New therapeutic strategies such as monoclonal antibodies act against different strains of multidrug resistant bacteria. Another area where focus should be made is in boosting host defence and innate immunity. There is a need to develop new or more effective immunisation against respiratory bacteria and viruses, particularly for the prevention of Respiratory syncytial virus (RSV) and Pneumococcal infections. Only a few newer formulations of antibiotics are in the pipeline for bacterial respiratory infections. The dramatic shortage of new antibiotics together with the increasing number of antibiotic-resistant bacteria is a worrying threat to the global population and are a critical challenge for health care institutes. Molecular bacteriology and molecular virology have to progress at a faster pace.

The future of Microbiology will determine what advances will be made in Respiratory Medicine.

\section{REFERENCES}

[1] Mizgerd JP. Acute lower respiratory tract infection. N Engl J Med 2008;358(7):716-727.

[2] Macfarlane JT. Acute respiratory infections in adults. Brewis RAL, Corrin B, Geddes DM, et al. (eds). Respiratory Medicine, London: W.B. Saunders Company LTD 1995.

[3] Mandell LA, Wundink RK. Harrison's text book of pulmonary and critical care medicine. Chapter 11, 2013:P 105.

[4] Dawadi S, Rao BS, Khan GM. Pattern of antimicrobial prescription and its cost analysis in respiratory tract infection. Kathmandu Univ J SciEng Technol 2005;1(1):1-93.

[5] Chapman S, Robinson G, Stradling J, et al. Lung abscess. Oxford handbook of respiratory medicine. Chapter 40, 2014:P 444.

[6] Erling V, Jalil F, Hanson LA, et al. The impact of climate on the prevalence of respiratory tract infection in early childhood in Lahore, Pakistan. J Public Health 1999;21(3):331-9.

[7] Murray CJ, Lopez AD. Mortality by cause for eight regions of the world: global burden of disease study. Lancet 1997;349(9061):1269-76.

[8] Ozyilmaz E, Akan OA, Gulhan M, et al. Major bacteria of community-acquired respiratory tract infections in Turkey. Jpn J Infect Dis 2005;58(1):50-2. 